\title{
Smell walking and mapping
}

Link to publication record in Manchester Research Explorer

\section{Citation for published version (APA):}

Perkins, C., \& McLean, K. (2020). Smell walking and mapping. In S. M. Hall, \& H. Holmes (Eds.), Mundane methods: Innovative ways to research the everyday (pp. 156-173). Manchester University Press.

\section{Published in:}

Mundane methods

\section{Citing this paper}

Please note that where the full-text provided on Manchester Research Explorer is the Author Accepted Manuscript or Proof version this may differ from the final Published version. If citing, it is advised that you check and use the publisher's definitive version.

\section{General rights}

Copyright and moral rights for the publications made accessible in the Research Explorer are retained by the authors and/or other copyright owners and it is a condition of accessing publications that users recognise and abide by the legal requirements associated with these rights.

\section{Takedown policy}

If you believe that this document breaches copyright please refer to the University of Manchester's Takedown Procedures [http://man.ac.uk/04Y6Bo] or contact uml.scholarlycommunications@manchester.ac.uk providing relevant details, so we can investigate your claim.

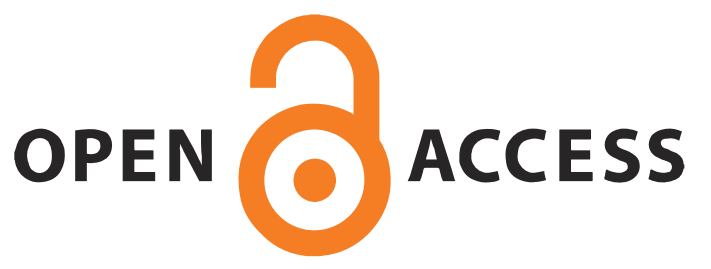




\section{AUTHOR QUERY FORM}

Dear Author,

During the preparation of your manuscript for publication, the questions listed below have arisen. Please attend to these matters and return this form with your proof.

Many thanks for your assistance.

\begin{tabular}{|l|l|l|}
\hline Query & References Query & Remarks \\
\hline
\end{tabular}

1 AUTHOR: This sentence seems incomplete. Please check and amend if necessary.

AUTHOR: "ref. McLean 2014" has not been cited in the text. Please indicate where it should be cited; or delete from the Reference List. 


\section{0}

\section{Smell walking and mapping \\ Chris Perkins and Kate McLean}

\section{Introducing the aroma}

Smell offers a ubiquitous and powerful way to make sense of the world and strongly underpins social hierarchies, working as a key cue in social bonding. Smells also have a strong cultural resonance. They take on different meanings in different contexts, changing over time and across cultures. The perception of smells powerfully evokes memories of experiences and emotions associated with events. As such, smell is inevitably mundane, quotidian and central to life.

However, smell as a sense is largely taken for granted and as such is under-analysed. Artistic practice has privileged vision over smell (Drobnick, 2002). To date most research on smell has been scientific and technical, focusing on psychological aspects of the sensory modality, or the neuroscience of perception, or the utility of scent development and commodification. As such, olfactometers can be deployed to measure environmental odours and pollution monitoring can be carried out. But for most people smells can be difficult to research: they are discontinuous, intangible, ephemeral or episodic. They can be pleasant as well as a nuisance. Smells are ingested: volatile molecules are inhaled and processed by the limbic system in the brain, whereas a landscape that is seen can be framed as separate from our corporeal being and as such more subject to reason. So, perceptions of smell are emotional, subjective and more separate from cognition, 
which makes them challenging to deploy in our methodological toolkits. Just as modern society has become increasingly sanitised, with smell kept in its undervalued place, so has research tended to underplay the multiple social, cultural and geographical roles that smell can play. Sight allows fixed perceptions of the world to emerge, be mapped and shared but the more mutable, contingent and ambiguous qualities of smell present interesting challenges for researchers.

It is these challenges involving juxtapositions of vision and smell that form the focus of this chapter. The geographies wrapped up with smell relate to our everyday experiences and the mapping of these perceptions and their affects has great potential for revealing hitherto unseen social and cultural norms. The mundane can become extraordinary when designers translate what Porteous (1985) called the 'smellscape' into visual forms and share these with others. Mapping offers a method that is particularly appropriate for achieving this. Maps have historically usually fixed the ambiguous or ephemeral, tying down meanings and freezing time and allowing a shared worldview to emerge as a rational working tool. However, recent technological and epistemological change has encouraged a focus on more performative and narrative qualities of the form (Perkins, 2009). So, the time is appropriate for increased encounters and translations between smell and maps (for further discussion on encounters see Tipper, this collection).

This chapter examines some of the background and ways in which artists, designers and researchers have enacted these encounters and translated between sensory modalities. It explores the challenges of smell-mapping practice. It charts some of the practical fashions in which smell mapping might be enacted, focusing upon different temporalities associated with our smellscapes, and in particular on ways of carrying out a smell walk and mapping smell. It documents the potential of different technologies and mobilities for attending to smell, highlighting different kinds of smell walking (other sensory forms of walking interviews are discussed by Rose, this collection). The links between smell and other sensory geographies are explored. In so doing, this chapter argues for a multi-sensorial turn in mundane methods.

\section{The background to smelly mapping methods}

In a ground-breaking review Porteous (1985) highlighted the marginalisation of senses other than vision and first developed the notion 
of the smellscape as a scaled, subjective assemblage of olfaction, people, contexts, histories and geographies; the olfactory equivalent of a landscape or soundscape. He flagged up the need for real-world investigation of smellscapes through what he termed 'smell walks', as against laboratory-based investigations. In the last decade of the twentieth century and the first two decades of the new millennium researchers have increasingly addressed the everyday aspects of smells in society and culture. Sensory studies has emerged in this period as an important cross-disciplinary field of interest to disciplines across the social sciences and humanities, with the journal Senses and Society available from 2006. Smelly research is much more on the agenda in 2019, with overview monographs in cultural history (Classen, Howes and Synnott, 1994); sociology (Low, 2008); social anthropology (Drobnick, 2006); and urban design (Henshaw, 2014) now situating smell in relation to their different concerns. Henshaw et al.'s (2017) collection drew authors from fields as diverse as museum curating, artistic practice, archaeology, history, landscape design, geography, psychology, literary studies, organisation studies, environmental management and education. Across these fields researchers are increasingly exploring the relations of smell to place.

However, this focus on smell has only rarely generated novel methods. Sarah Pink's (2015) overview of sensory methods charts a very wide variety of methodological innovations in everyday geographies. But her consideration of smell is very much in terms of its potential to elicit participation in conventional ethnographic methodologies. It is certainly true that most methodological work with smell focuses on environmental monitoring of odours or air quality, as part of strategies to manage nuisance, and are very much the domain of environmental consultancies and specialist technical equipment. Social scientific methods charted by Pink do incorporate smell into interviews, or focus groups, but do not directly attend to smell. By way of contrast to technical assessments, or smells' subsidiary role in other ethnographic approaches, this chapter focuses upon the creative deployment of mapping as a mobile method, building on Porteous's original suggestion and Henshaw's (2014) development of the concept, and drawing in particular on the work of one of the authors, sensory designer Kate McLean.

Mapping as a process begins with the planning of a strategy, incorporating thinking as well as doing. It reflects a research design relating 
to collecting information to be mapped and decisions about what and how to map. The mapping helps navigation, or is used to administer and control, or it focuses on distributions and relationships, or on a specific aspect of a place. It might map out a view of the past, imagine a future or chart something happening now. It might be an information source or serve as part of persuasive narrative. Maps can stand on their own right or be designed as part of a wider assemblage. They can serve as part of a neutral discourse or be strongly crafted to evoke particular emotional responses about a place as an artwork, a promotional device or as tools in a subversive protest. The poetics of a design come together with a political context. Mapping has historically been associated with facts, with best practice in cartographic enterprise and with the power of the nation state. But the aesthetics of mapping highlight interpretive and subjective qualities, and at the same time mapping also reflects and enrols people as a social practice. Mapping technology has profoundly impacted practice. Digital developments and the social network have removed past certainties and opened new opportunities for anyone wishing to map, arguably democratising the medium.

In the light of this complexity it makes sense to recognise that smell mapping becomes a performance that changes depending on the stage of mapping. On the one hand, it might involve the synthesising of smells - as in the work of Sissel Tolaas, who incorporates distilled essences of mundane smellscapes into exhibition spaces, such as in her 2012 work SmellScape KCK/KCMO (Lockard, 2013). On the other hand, there are published maps of smellscapes that seek to depict the olfactory environment by translating smell into visual equivalents. The history of publication of this kind of mapping has until recently focused upon the final stages of the process - the design of a map to depict a smellscape. Among design challenges that have to be addressed are how to classify smells, how to represent their intensity and how to deal with the transience of the smellscape. There is no published consensus on any of these issues but some of the practical issues relating to these design concerns are explored in more detail in the next section. Three methodological innovations have been significant in recent smell mapping.

The nature of data collection has changed in profound ways as a result of the capacity of social networking to crowdsource the collection of smells. Big data can be repurposed to map the smellscape. 
Quercia et al. (2015) highlight this potential in their discussion of the scraping and subsequent mapping of geo-referenced picture tags from Flickr and Instagram and geo-referenced tweets from Twitter, and argue that this can allow an upscaling of data collection. The researchers derived a tenfold classification of smell tags, across two test data sets relating to the cities of Barcelona and London. Thus it became possible to map the base notes of smell for different cities: Barcelona was characterised by smells relating to food and nature, whereas London was represented predominantly by smells relating to traffic emissions and waste. The mid-level notes of the smellscape, with a finer spatial definition, can be displayed as heat maps in a mash-up against a map of the street segments to which the terms might apply. Borough Market in London is associated with high scores in posts relating to the twenty-four-hour city, with the smells of leisure and entertainment dominating. By way of contrast, high levels of posts relating to pollution cluster along significant roads across the capital.

Another data collection technology that is profoundly impacting smell collection is potentially driven by using mobile devices and customised apps to automate the smell-mapping production process. Apps such as Smell PGH allow posts about local perceptions of unpleasant smells. Prototypes of several different systems have already been designed to extend this idea to the wider range of smells, including the Smellscaper App from Kate McLean, which automates the smell-noting process described below.

Digital mapping also allows many different subsequent aspects of the mapping process to be automated. The fixed framing of the hard copy map is no longer a constraint. The angle of view onto a smell map can be altered, to convey different impressions of the data. McLean (2018) reports on changing the viewing angle in an animated smell map of Pamplona. A bird's-eye and top-down perspective (referred to as planimetric) allows the appearance of a smell to be charted. A view from above, at 45 degrees (known as an isometric perspective), allows dissemination of smells in the wind to be charted and maps changing durational perceptions of the smellscape. And a more immersive angle of view, moving through a smellscape in a horizontal fashion, charts the volatisation of smells as a person encounters an aroma that subsequently disappears. The technology enacting the display alters the impression. Models of smells diffusing can be simulated in exhibition displays. Digital animation can convey durational qualities of 
the smellscape. Display technology alters the interaction that might be possible. A map on a mobile device affords readers with many tasks that are beyond the fixed paper maps, such as panning, zooming and moving as the device itself moves through the smellscape, but even though the display may be egocentric, with the map moving as the reader moves, overview is limited by the screen size of the display.

So, the design choices around the mapping of smell are complex and dictated by contextual factors such as the environment, the temporality of the smellscape, the nature of mapping technologies, the desired impression for reading, and more pragmatic issues such as medium of dissemination and resources available to the designer or researcher.

\section{Smell walking and mapping in practice}

This section explores some of the key practical issues that underpin mapping out smells, drawing on Kate McLean's artistic practice. Smell mapping entails many different activities: collecting smells, classifying them, representing them in mapped form and then displaying a map in different contexts. At each of these stages different configurations are possible and we highlight below the potential of smell walking as a data collection strategy; practices of map design; the multiple views of the smellscape that can be made; and the exhibition contexts in which smell maps have been displayed.

It has been argued that the smell walk is an essential initial step in the mapping of the smellscape which can serve as a useful real-world strategy for collecting sensory perceptions about a place (Porteous, 1985). It is now widely accepted that smell walking offers an active, researcher led, embodied methodology with the capacity to attend to more than vision and more than representation (for further embodied methods see Hall et al., Collins, and Tipper, this collection). Best practice in smell walking very much depends upon the kind of smell walk that is undertaken and McLean (2019) describes five different variations, according to the number of participants, the degree of expert participation and the use of different data collection technologies: she identifies the solo walk, the group walk, the buddy walk, the 'smellfie' and the app walk. Buddy walks enrol a local expert who knows the smellscape and is able to lead the researcher to the best locations. App walks deploy digital technology, instead of printed forms, 
to record smell notes. Smellfie walks are a kit that may be used by anyone to set up a smell walk instead of relying on a guided approach.

Between July 2011 and August 2018133 different smell walks enrolling around 1,200 participants were led by one of the authors of this chapter as part of her ongoing doctoral research (McLean, 2019). The majority of these took place in urban contexts, where smell diversity might be expected to be greater because of the greater diversity of human activities, and in European or North American cities. Early walks were solo and during this process McLean developed best practice methods for different stages of the activity. In part they were a learning process for the researcher and changes in walking strategy reflected learning from failure. For example designing and using technology in app walks was initially very appealing, but this kind of walk was largely abandoned after 2017. This was mainly because using a mobile device to record smells distanced walkers from the world by demanding attention be given to the screen, and the app also discouraged discussion (for further walking-based methods see Rose, this collection).

Indeed discussion emerged as important in the process and the group walk has been the most common form of smell walking - ninety-five of the walks have deployed this format. Group walks allow the researcher to enrol many noses as sensors, and cover a much wider area, but also bring together sometimes-contested views of the smellscape. Practical advice about smell walking is described in the next section.

The design of mapping produced during this sensory work always uniquely reflects the particular smellscape. Practice usually takes many people's perceptions and translates these into maps - so the mapping is predominantly an artistic and phenomenological recounting of multiple sensory experiences, a creative re-mapping that speaks to aspects of a unique place experienced at a particular time.

The maps emerge from a wide variety of smells accumulated during smell walks. Smells are classified from perceptions during the smell walking. The Amsterdam map identifies 11 smells distilled from 650 smell perceptions. These classifications frequently evoke different notions of place, and do not always conform to expected stereotypes. Thus SmellMap Amsterdam does not record cannabis, which was only noticed in a few neighbourhoods, but instead more frequently records waffles, spicy food, floral scents, coffee and old books, set against the damp and all-pervading base notes of the canals (McLean, 2017). In other contexts a very different coded range of smells might be mapped. 
In the Newport, Rhode Island map, for example, maritime smells dominate, whereas the 'scent mapping' of Singapore - so designated because of funding - reflects much more of an emphasis on food.

Many of these maps are designed in a consistent style, characterised by colour-coded points indicating where smells were perceived, alongside concentric circles denoting potential dispersal from these sources. Perceived smell intensity and wind strength and direction come together in the characteristic contoured patterns. The maps deploy pastel tones describing smells, which are set against restrained and frequently limited base information. The published maps do not capture the smellscape in a scientific way; instead they are akin to what landscape architect James Corner (1999) terms 'agentic mappings', emerging out of individual creative moments, but with the power to change perceptions of places. So, the published smell map becomes part of a narrative where the designer controls the final published output, while acknowledging the many noses that have come together in the process. These social or group perceptions of the smellscape are set against interpretation from the artist.

However, publication is only one of many aspects of mapping. Many of the maps are designed to facilitate the smelling process, such as route maps for a group smell walk. Or they exist as working diagrams, to be changed by the artist or researcher as the process crystallises. Some maps explicitly chart the dynamism of the smellscape, evoking aspects of the temporality of smell (McLean, Lammes and Perkins, 2018). They can indicate the duration of a smell, the tempo at which the smellscape changes, the sequence of encountering different smells and then losing them in the course of a walk, and the more rhythmic qualities of the smellscape. Mapping of Kyiv carried out by McLean in the winter of 2017 and emerging from group walks through the city, for example, highlights many different ways in which temporal qualities of the smellscape might be mapped out (McLean, 2019).

Published mapping is frequently displayed in exhibition spaces as part of a commissioned outcome. In some of these exhibitions maps serve as props to encourage participation from audiences. For example the Marais (Figure 10.1), Amsterdam and early Parisian maps were exhibited alongside representative smells derived from natural and synthetic sources, and visitors to the exhibitions were encouraged to sniff the cases and at times to post their own reactions to the 


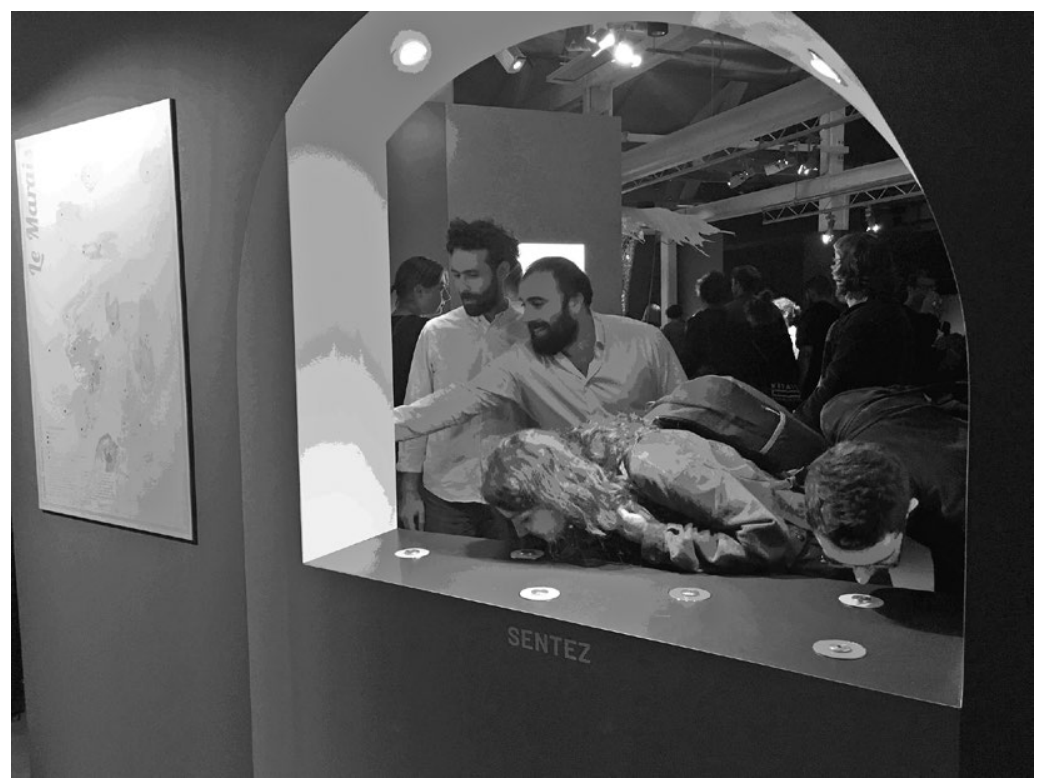

10.1 Smellmap Le Marais (2018) exhibited at MAIF Social Club, rue de Turenne, Paris comprises two versions of the visual smell map on either side of a wall and sniffing bottles (hidden beneath a surface) containing essential oils and raw materials of the featured and mapped smells of bamboo, leather, painter's varnish, perfume, peach and wood

distillations against the visual representation of the smellscape, adding further layers to the complex exhibited assemblage.

Versions of the published mapping are also disseminated from the http://sensorymaps.com/ website. For the majority of the walks, however, mapping may not actually be created. So, the practicalities of smell walking and smell mapping very much depend on the priorities of researchers or practitioners deploying the method. We conclude this section by contrasting McLean's practice with research from different disciplines and contexts.

The strategy of smell walking and mapping can be deployed as part of many different inquiries in different kinds of spaces. The emotional correlates of smell strongly suggest that the technique has great potential in charting cultural geographies. It can be used by sociologists to 
map the spread of gentrification, for example by mapping out the distribution of beard oil and sourdough baking around markets in the east end of London (Rhys-Taylor, 2018). Historians can re-create past sensory geographies of cities, as evidenced for parts of Istanbul by Davis and Thys-Şenocak (2017). Smell can also be deployed as an active part of storytelling, to evoke emotional responses to place and unlock memories. But conversely it can also be used to chart the marketing of the city, highlighting ethnic districts which trade on the back of their smellscapes, as described, for example, by Henshaw (2014) in relation to Manchester's Chinatown. A more corporeal approach can document smell to explore the affect of the office as a place of work, as described by Riach and Warren (2015). Planners and landscape professionals can use smell walking as a means of investigating how a smellscape contributes to perceptions of pleasantness, such as in the work of Xiao, Tait and Kang (2018). The mapping of the smellscape can also directly contribute to multi-sensory urban and landscape design of urban greenspaces (Kang, Tait and Xiao, 2017). Smell walking and mapping can also be used in pedagogy, as part of student-led field investigations, focusing on the embodied and social practices through which we encounter places, but also on the methodological differences that stem from multi-sensory encounters (Phillips, 2015; Playful Mapping Collective, 2016).

So, a human-centred and graphic design-oriented creative mapping offers only one way in which smell walking and mapping might be deployed. The potential is there for a wider uptake, and for following best practice in deploying the method.

\section{Advice for others}

Graphical ability, research skills and research questions strongly impact upon what might be best practice. However, in this section we focus on two key aspects: how to set up a successful smell walk, and how to design a smell map to convey perceptions of a smellscape to an appropriate audience. Kate McLean has provided a kit comprising guidance and inviting independent investigation by anyone using smell walks as a starting point for mapping the smellscape. This is supported by online documentation available at sensorymaps.com/ wp-content/.../10/Smellwalk_Intro_Kit_CKateMcLean_2015.pdf As su and is accompanied by videos explaining the process of enacting 


\begin{tabular}{|c|c|}
\hline \multicolumn{2}{|c|}{ Box 10.1: Best practice in smell walking } \\
\hline $\begin{array}{l}\text { Phases and } \\
\text { timing }\end{array}$ & $\begin{array}{l}\text { Maximum forty-five minutes' walking time: nasal } \\
\text { attention wanes } \\
\text { Start with smell catching } \\
\text { Then smell hunting } \\
\text { Then free smelling }\end{array}$ \\
\hline Route & $\begin{array}{l}\text { Anticipate appropriate variety of natural and synthetic } \\
\text { smells to maintain interest. Provide a simple } \\
\text { suggested route }\end{array}$ \\
\hline Group size & $\begin{array}{l}\text { Up to twelve people is ideal: if larger numbers then } \\
\text { separate into sub-groups }\end{array}$ \\
\hline $\begin{array}{l}\text { Nose training } \\
\text { and practice }\end{array}$ & $\begin{array}{l}\text { Encourage discussion between group members after } \\
\text { each stage is completed } \\
\text { Drink water to improve smell capability } \\
\text { Sniff own skin for relief to 'reset' nose }\end{array}$ \\
\hline Smell notes & $\begin{array}{l}\text { Introduce participants to deliver appropriate and } \\
\text { consistent smell notes either in hard copy or in } \\
\text { digital form } \\
\text { Record location: points but also lines and areas } \\
\text { Free text naming of smells } \\
\text { Numeric grading of perceived intensity } \\
\text { Numeric grading of perceived duration } \\
\text { Numeric grading of affect. Like / dislike } \\
\text { Expectations - expected or not } \\
\text { Free text association of personal feelings about smell }\end{array}$ \\
\hline $\begin{array}{l}\text { Post-walk } \\
\text { discussion }\end{array}$ & $\begin{array}{l}\text { Half an hour to discuss differences, trajectories and } \\
\text { rhythms and to reflect on base notes of the } \\
\text { smellscape with creative mapping exercise }\end{array}$ \\
\hline
\end{tabular}

a 'smellfie' (see https://vimeo.com/smellmap). This advice is summarised in Box 10.1.

Publicity is important for recruiting participants - a diverse group can work just as well as a narrowly defined demographic. Motivation is the most important factor in participation. Routes need careful selection, and should offer sufficient variety of natural and synthetic smells to keep the attention of participants, but walks should not last 
too long. Beyond forty-five minutes it becomes harder to maintain motivation to note smells, because of the unusual concentration required to attend to what our noses detect. Weather conditions strongly influence smellscapes: a windy day will disperse smells further from their sources; a humid and still day can enhance the richness of the smellscape; on a warm day walkers are likely to perceive different odours from those smelt during a cold walk. So, expect different outcomes on different days.

Setting up the walk is important. Material should be given out on which participants can record their reflections in the form of 'smell notes', and a mix of quantitative and qualitative evidence works best (see Box 10.1). In most cases this will involve manual note taking but apps to automate procedures are likely to become more available. Be aware that use of an app focuses attention away from physically detecting smells. A map of the recommended route can also be provided for participants. Practical advice needs to be given, about risks, ethics and how to take smell notes. During the walk it is helpful to pay attention to three different kinds of smell:

- curious or unexpected smells that are short-lived, and which will be individually noted, such as perfume on a passer-by or woodwork being painted. In an analogy to perfumiers' use of smell, these volatile odours form the top notes of a smellscape;

- episodic elements of a smellscape reveal specific local areas of a town, such as the smell of fish from a market, or fried food from a takeaway - analogous to the middle notes; and

- background smells that form a context and a constant element in the smellscape, for example the residual smell of a brewery, or the dampness of a canal, which make up the base notes of the smell pyramid.

It is suggested that small groups work best - up to twelve people talking about the smells they encounter encourages creative social reflection and brings together different opinions about the smellscape. Individuals walking alone will miss smells, and larger group sizes can distract. Smell-walking practice is best enacted in different phases. Smell catching or passive smelling involves walking slowly through an area focusing on smell as the primary sense - breathing in deeply - and attending to the aromas that are encountered. This is a good strategy for starting a smell walk. A more active phase can then follow once participants have 


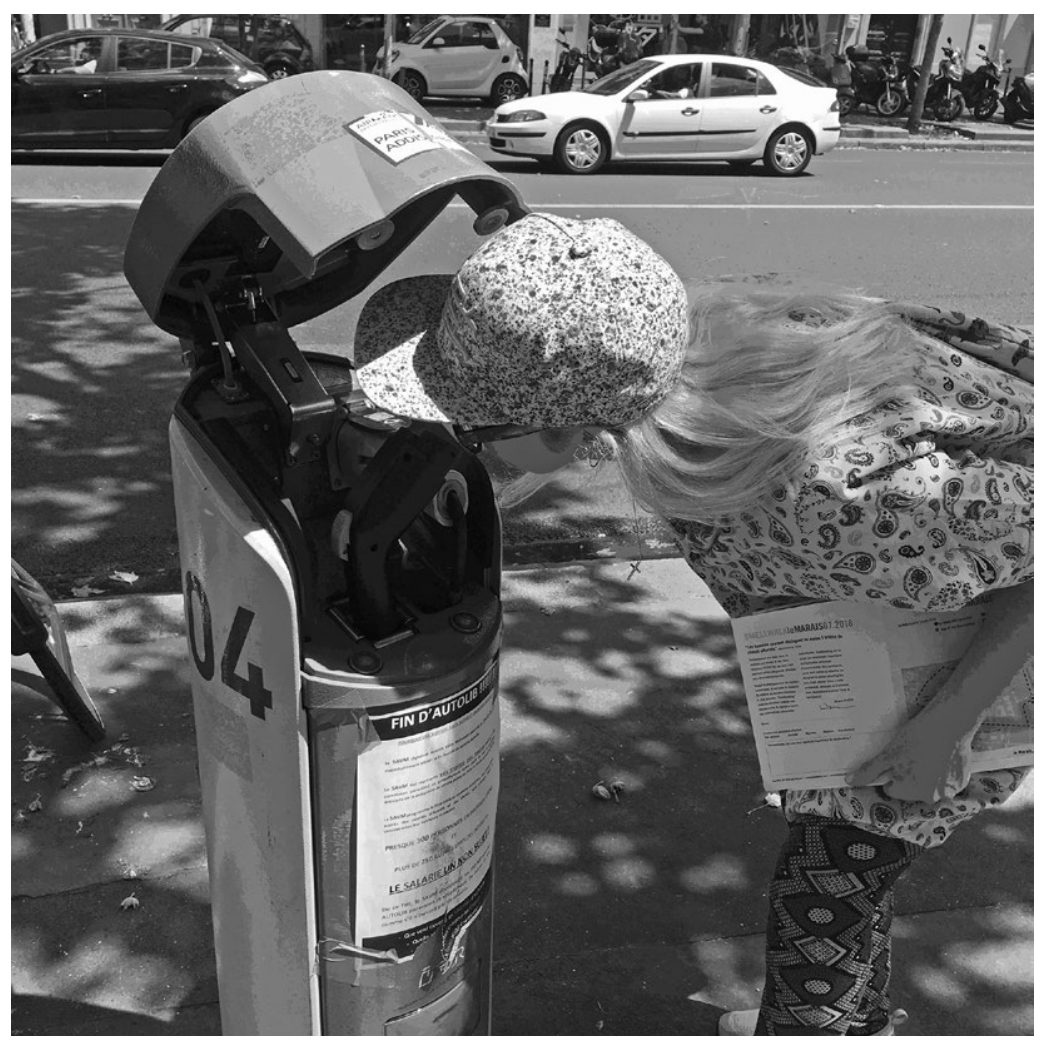

10.2 Smell walk participant holds her smell notes as she explores the Marais area of Paris, anticipating the potential smells of a recharging unit

got used to the process. Smell hunting involves seeking hidden smells, by using other senses to hunt them down, anticipating likely associations such as the smells around a litter bin, or taking action to make a smell, such as crushing leaves (Figure 10.2). Free smelling works well as the final phase of a smell walk, and keeps participants engaged in the process. A post-walk discussion offers a useful way to bring the experience to fruition, with talk starting from smell notes made during the walk. Smell sketching can also be a useful way for individuals to situate their own olfactory experience (for further discussion on sketching as a method see Heath and Chapman, this collection). Out of this shared group experience different individual perceptions of the smellscape 


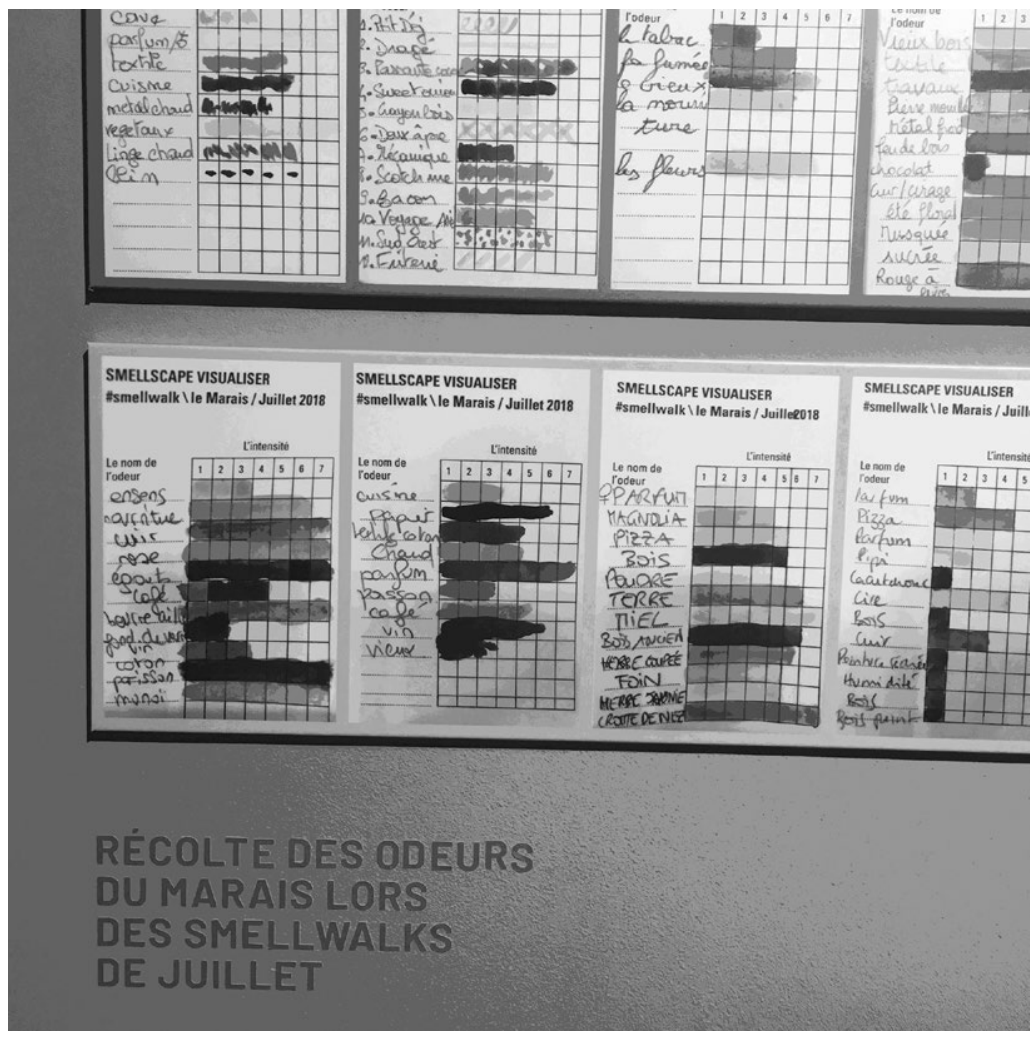

10.3 Detail from 'Smell Harvest from the Marais during July Smellwalks' (2018)

emerge. The smellscape visualiser graphics shown in Figure 10.3 were completed by different smell walkers immediately following their smell walk and demonstrate this variety of individual perceptions of a shared route as well as similar smell experiences. Each walk is displayed as a horizontal set and the different colours deployed are a good way to creatively represent individual smell associations.

These smell-walking outcomes can be mapped by individual participants in a workshop or by the researcher coordinating the smell walks after the event. For a researcher with limited graphical skill it makes sense to work through the multiple visualisations documented at http://'maps. $\mathrm{com} / \mathrm{so}$ as to be aware of possibilities, but also to seek advice about 


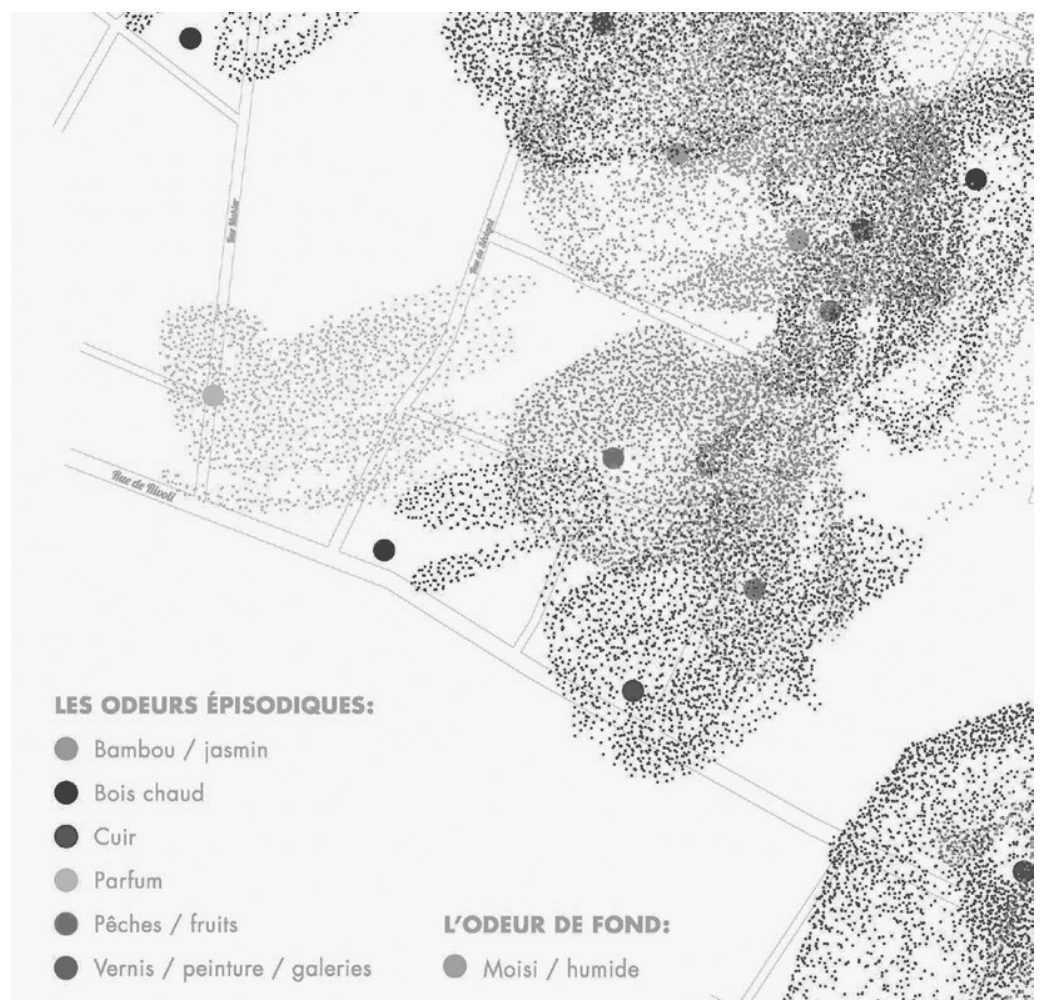

10.4 Detail from 'Smellmap: Le Marais' (2018)

graphic design in guides such as Perkins (2016) or Wood and Krygier (2016). If in doubt keep the maps as simple as possible, using a graphic variable such as colour hue to discriminate between different smells, and making sure that an appropriate visual hierarchy is maintained with any base information working as the ground to enhance interpretation of the smells that serve as the figure. Figure 10.4 illustrates Kate McLean's design practice and her clear use of the visual hierarchy of colour between background and smells, as well as a systematic approach to icon design in which a large dot indicates a smell source and smaller dots allude to scent molecule dissipation in wind conditions noted on the days of the smell walks. However, it is important to be very clear of the intended audience. Think carefully about any mapping of change, and perhaps deploy juxtaposition of multiple maps to convey impressions of mutability. Choose 
a scale that is appropriate for the amount of detail. If exhibiting maps, make sure that the mapping clearly relates well to underpinning narratives of the research.

\section{Conclusions}

By focusing attention onto our noses as against our eyes we can begin to notice the everyday information that they collect, and in so doing reassert the importance of senses beyond vision. Smell as something beyond cognition is a sense that speaks directly to a more embodied approach to mundane experience, and as such smell mapping can tell different stories about place from those narrated in methodologies more anchored to sound and vision. As such a more than representational appreciation of places and corporeal experience can usefully be informed by deploying smell mapping as a method.

In this chapter we have argued that the smell walk is a mobile method that offers a useful way of delivering smell mapping, generating a systematic and different appreciation of our everyday experiences. The smell walk, then, can generate different views of space; maps that focus on the intangible and ephemeral instead of the material and fixed (McLean, 2018). Making maps from the shared and contested experiences of smelly places can allow these qualities to be shared. A rigorous application of the methods described above shows the olfactory diversity that still survives and illustrates the importance of Porteous's (1985) argument for attending to the real-world qualities of the smellscape. It shows how smell can escape the specialist laboratories of the perfumier to become part of geographers' phenomenological methodological armoury. By following up on Henshaw's and McLean's work, and in particular the sources outlined in Box 10.2, a richer appreciation of everyday life becomes much more possible.

\section{Box 10.2: Further reading and useful resources}

Henshaw, V. (2014) Urban Smellscapes: Understanding and Designing City Smell Environments, London: Routledge.

McLean, K. (2015) Smell walk introductory kit, www.sensorymaps.com/ wp-content/.../10/Smellwalk_Intro_Kit_CKateMcLean_2015.pdf (accessed 27 September 2019). 
The smellscape is taken for granted in our culture, but is itself a frequently unnoticed outcome of capitalist accumulation. As such, smell is a commodified part of a global system. Western cities are increasingly bland and inoffensive spaces in terms of their smells - a significant change from the richly offensive and diverse sensory experiences offered by these places in the past, and which still characterise slums and many parts of cities in the global South. So, a smell walk can become part of a political movement to reassert the importance of local diversity in our smellscapes, and the mapping of smells accumulated during smell walks can serve as a mechanism for telling different stories about these places, beyond the corporate blanding of global retail centres. By searching out olfactory difference and deploying maps to share this with others we can register the importance of a smell heritage that risks being marginalised. And by smell walking we can make our methodologies more like embodied real life.

\section{Bibliography}

Classen, C., Howes, D. and Synnott, A. (1994) Aroma: The Cultural History of Smell, London: Taylor \& Francis.

Corner, J. (1999) 'The agency of mapping: speculation, critique and invention', in D. Cosgrove (ed.) Mappings, London: Reaktion Books, 213251.

Davis, L. and Thys-Şenocak, L. (2017) 'Heritage and scent: research and exhibition of Istanbul's changing smellscapes', International Journal of Heritage Studies, 23 (8): 723-741.

Drobnick, J. (2002) 'Toposmia: art, scent, and interrogations of spatiality', Angelaki: Journal of Theoretical Humanities, 7 (1): 31-47.

Drobnick, J. (2006) The Smell Culture Reader, Oxford and New York: Berg.

Henshaw, V. (2014) Urban Smellscapes: Understanding and Designing City Smell Environments, London: Routledge.

Henshaw, V., McLean, K., Medway, D., Perkins, C. and Warnaby, G. (2017) Designing with Smell: Practices, Techniques and Challenges, London: Routledge.

Kang, J., Tait, M. and Xiao, J. (2017) 'The design of urban smellscapes with fragrant plants and water features', in V. Henshaw, K. McLean, D. Medway, C. Perkins and G. Warnaby (eds) Designing with Smell, London: Routledge, 83-95.

Lockard, B. (2013) 'Sissel Tolaas, SmellScape KCK/KCMO', Senses and Society, 8 (2): 245-250.

Low, K. E. Y. (2008) Scent and Scent-sibilities: Smell and Everyday Life Experiences, Cambridge: Cambridge Scholars Publishing. 
McLean, K. (2014) 'Smell map narratives of place - Paris', NANO: New American Notes Online, 6, https://nanocrit.com/issues/issue6/smell-mapnarratives-place-paris (accessed 27 September 2019).

McLean, K. (2017) 'Smellmap: Amsterdam - olfactory art and smell visualization', Leonardo, 50 (1): 92-93.

McLean, K. (2018) 'Mapping the invisible and ephemera', in A. J. Kent and P. Vujakovic (eds) The Routledge Handbook of Mapping and Cartography, London: Routledge, 509-515.

McLean, K. (2019) Nose First: Practices of Smellwalking and Smellscape Mapping. PhD thesis, Royal College of Art, London.

McLean, K., Lammes, S. and Perkins, C. (2018) 'Mapping the quixotic volatility of smellscapes: a trialogue-interview with Kate McLean', in S. Lammes, C. Perkins, A. Gekker, S. Hind, C. Wilmott and D. Evans (eds) Time for Mapping, Manchester: MUP, 50-90.

Perkins, C. (2009) 'Performative and embodied mapping', in N. Kitchin and R. Thrift (eds) International Encyclopaedia of Human Geography, London: Elsevier, 126-132.

Perkins, C. (2016) 'Mapping and graphicacy', in N. Clifford, M. Cope, S. French and G. Valentine (eds) Key Methods in Geography (3rd edn), London: Sage, 598-619.

Phillips, R. (2015) 'Playful and multi-sensory fieldwork: seeing, hearing and touching New York', Journal of Geography in Higher Education, 39 (4): 617-629.

Pink, S. (2015) Doing Sensory Ethnography (2nd edn), London: Sage.

Playful Mapping Collective (2016) Playful Mapping: Playing with Maps in Contemporary Media Cultures, Amsterdam: Institute of Network Cultures, http:// networkcultures.org/wp-content/uploads/2016/12/PlayfulMapping InTheDigitalAge.pdf (accessed 27 September 2019).

Porteous, J. D. (1985) 'Smellscape', Progress in Human Geography, 9 (3): 356-378.

Quercia, D., Schifanella, R., Aiello, L. M. and McLean, K. (2015) 'Smelly maps: the digital life of urban smellscapes', International AAAI Conference on Web and Social Media (ICWSM), Oxford, 26-29 May.

Rhys-Taylor, A. (2018) 'Coming to our senses: multiculturalism, urban sociology and "the other" senses', in S. Nichols and S. Dobson (eds) Learning Cities: Multimodal Explorations and Placed Pedagogies, Singapore: Springer Singapore, 23-36.

Riach, K. and Warren, S. (2015) 'Smell organization: bodies and corporeal porosity in office work', Human Relations, 68 (5): 789-809.

Wood, D. and Krygier, J. B. (2016) Making Maps: A Visual Guide to Map Design for GIS (3rd edn), New York: Guilford Press.

Xiao, J., Tait, M. and Kang, J. (2018) 'A perceptual model of smellscape pleasantness', Cities, 76: 105-115. 